\title{
Longitudinal Count Data Analysis of Factors Affecting Epileptic Seizure of Patients in Case of Gondar Referral Hospital, Northwest Ethiopia
}

\author{
Belema Hailu Regesa*, Gizachew Gobebo Mekebo \\ Department of Statistics, Ambo University, Ambo, Ethiopia \\ Email address: \\ beellamaahailu@gmail.com (B. H. Regesa),gizmake@gmail.com (G. G. Mekebo) \\ ${ }^{*}$ Corresponding author
}

To cite this article:

Belema Hailu Regesa, Gizachew Gobebo Mekebo. Longitudinal Count Data Analysis of Factors Affecting Epileptic Seizure of Patients in Case of Gondar Referral Hospital, Northwest Ethiopia. American Journal of Bioscience and Bioengineering. Vol. 8, No. 4, 2020 , pp. 59-69. doi: 10.11648/j.bio.20200804.11

Received: June 4, 2020; Accepted: June 18, 2020; Published: July 28, 2020

\begin{abstract}
Even though the world is fighting epileptic seizure disease in unity and patients are getting treatment, it continued to be a serious health issue for parts of the world and a large number of patients are being registered every year. The main objective of this study was to identify associated risk factors affecting the progression of patients in Gondar Referral Hospital. In this longitudinal count data analysis, data was collected from 337 epileptic seizure patients registered for treatment from January 1, 2016 to April 30, 2018 in the Hospital and Poisson, Poisson-gamma, Poisson-Normal and Poisson-Gamma-Normal models were applied to the data. Poisson-Gamma-Normal model with random intercept was selected as a best model to fit the data based on different model selection criteria. The findings of the study revealed that time, brain injury, treatment, interaction of time with residence and interaction of time with brain injury were significant factors for epileptic seizure of the patients. Minimization of epileptic seizure of patients in response to treatment was observed, which means the patients were at decreased epileptic seizure when enrolled for treatment. Therefore, patients should be encouraged to stay on treatment.
\end{abstract}

Keywords: Epilepsy, Longitudinal Data Analysis, Seizure, Poisson-Normal Model, Poisson-Gamma-Normal Model

\section{Introduction}

Epilepsy affecting approximately 69 million people worldwide and the majority of whom $90 \%$ lives in resourcepoor countries [1]. Epileptic seizures are manifested by an abnormal, excessive, and hyper synchronous electrical discharge of neurons in the brain. Seizure can cause changes in behavior, movements or feelings, and in levels of consciousness. Each distinct form of epilepsy has its own natural history and response to treatment. This diversity probably reflects the many different underlying causes of epilepsy and the variety of epilepsy syndromes in which the clinical and pathological characteristics are distinctive and suggest a specific underlying etiologic mechanism. There are many kinds of seizures, each with characteristic behavioral changes and electrophysiological disturbances that can usually be detected in scalp electroencephalographic (EEG) recordings. A person is considered to have epilepsy when two or more unprovoked seizures occur that can't be explained by a medical condition such as fever or substance withdrawal. Seizures can be the result of a family tendency toward the disease, or they can occur after a brain injury, but the cause of epilepsy is largely unknown [2].

Patients with epilepsy have poor health outcomes, including greater psychological distress, depression, anxiety, employment restriction, more physical injuries such as fractures and burns, and increased mortality, besides epileptic seizures result in devastating social consequences which result in poor quality of life. When the number of seizure frequency increases, depression and perceived stigma also increase which further results in the devastating social consequences and complication of patients' quality of life. Seizures can last from a few seconds to a few minutes. Patients and health care professionals do not always 
recognize the signs or symptoms, which can include convulsions, a loss of consciousness, blank staring, lip smacking, or jerking movements of the arms and legs. A seizure has a clear beginning, middle, and end. A seizure is a transient epileptic event, indicating a disturbance in brain function. Having a single seizure does not necessarily mean that a person has epilepsy [3].

The prevalence of epilepsy in Africa ranges from 2.2 to 58 per 1000 population and it is the second or third most common reason for consultation and hospitalization. In Ethiopia, review of community-based epidemiological studies of neurological disorders showed that epilepsy is a prevalent neurological disorder and the prevalence of the disease in the country was reported to be 5.2/1000 inhabitants at risk with the annual incidence of 64 in 100,000 inhabitants. A Cross-sectional study in Gondar was done on 354 adult epileptic patients and a total of 79 adverse events were reported. The most frequently encountered adverse events were fatigue, gastrointestinal disturbance and sedation/depression [4].

Shortness of medical services, unavailability of antiepileptic medications, and lack of awareness of medical treatment and cultural-related factors are more common phenomena in developing countries including Ethiopia [5]. WHO, the International League Against Epilepsy (ILAE) and the International Bureau for Epilepsy (IBE) have led the Global Campaign Against Epilepsy to bring the disease "Out of the Shadows" to provide better information and raise awareness about epilepsy and to strengthen public and private efforts to improve care and reduce the disease's impact [6].

Identifying the risk factors of epilepsy is essential to inform public health policies so as to design strategies that can improve care to epilepsy patients and reduce the impact of the disease to improve the patients' health and quality of life. This study, therefore, has tried to fill the gaps in understanding the status of epilepsy patients by identifying determinant risk factors of epilepsy in Gondar Referral Hospital.

Generally, this study has attempted to answer the following basic research questions:

a) Which factors significantly affect the epileptic seizure of patients?

b) What is the progression of epileptic seizure of the patients over time?

c) What is the effect of the treatment given to the patients? d) Which model is the most appropriate for analyzing the predictors of epileptic seizure of patients?

Objectives of the study

The main objective of this study was to investigate factors that affecting the epileptic seizure of patients in the case of Gondar Referral Hospital. More specifically:

a) To identify the significant factors that affect epileptic seizure of the patients.

b) To explore progression of epileptic seizure of the patients over time.

c) To identify the effect of treatment given to the patients.

d) To choose appropriate model that can fit epileptic seizure of the patients.

\section{Methodology}

\subsection{Study Area Description}

This study was conducted in Gondar in Gondar Referral Hospital. Gondar is a self-governing city administration along with those 22 woredas found in north Gondar administrative zone which is one of the eleven zones in the Amhara national regional state of Ethiopia. Gondar is located at a distance of $750 \mathrm{Km}$ far from Addis Ababa, the capital of Ethiopia. In Gondar city, there are also many health institutions that are providing health service to the population of which the largest one is Gondar Referral Hospital. Apart from all other health service, the hospital is serving large number of epileptic seizure patients.

\subsection{Data Source and Sample Size}

For this study secondary data were used. The data were obtained from epileptic seizures patients' documents at Gondar Referral Hospital from January 1, 2016 to April 30, 2018. A total of 337 epilepsy patients were included in the study. The data analysis was done using Rstudio and SAS (version 9.4).

\subsection{Variables in the Study}

\subsubsection{Response Variable}

The response variable of this study is epileptic seizure of patients.

\subsubsection{Explanatory Variables}

The explanatory variables which are supposed to influence the epileptic seizure patients are given in the Table 1 below.

Table 1. Predictor variables with descriptions and codes.

\begin{tabular}{lll}
\hline Variables & Description & Categories(if any) \\
\hline Age & Age of the patients & Continuous \\
Gender & Sex of the patients & $0=$ "Female", 1= "Male" \\
Residence & Residence of the patients & $0=$ "rural", $1=$ "urban" \\
Physical exercise & Regular exercise of the patients & $0=$ "No", 1= "Yes" \\
Time & Time to visit of the patients & $0=$ "first visit", 1= "second visit", 2= "third visit", 3= "fourth visit" 4= "Fifth visit", 5= \\
Baseline & First visit of the patients & $0=$ "fixth visit", $6=$ "Seventh visit" \\
Treatment & Treatment given for the patients & $0=$ "Phenobarbital", 1= "Phenytoin", 2= "Others" \\
Family history & Family history of the patients & $0=$ "No", 1= "Yes" \\
Brain injury & rain injury of the patients & $0=$ "No", 1= "yes" \\
Educational level & Educational status of the patients & $0=$ "No formal education", $1=$ "Primary", 2= "Secondary", 3= "Above" \\
\hline
\end{tabular}




\subsection{Method of Data Analysis}

\subsubsection{Longitudinal Count Data Analysis}

A longitudinal study is a prospective study where repeated measures are taken over time for each individual. Longitudinal data are universal in a wide range of fields: medicine, public health, education, business, economics, psychology, biology and more. Longitudinal data analysis is a statistical analysis method in which the variable of interest (the dependent variable) is measured repeatedly over time and the repeated measurements taken from a subject over time are correlated. The distribution of the response variable in longitudinal data analysis may be Gaussian or nonGaussian. The Gaussian longitudinal data are often analyzed by linear mixed model [7] and generalized linear mixed model is the most frequently used mixed effects model in the context of discrete or non-Gaussian longitudinal data.

In practice, it is common to face response variables of count type like epileptic seizure and number of CD4 cells in a cubic milliliter of blood. Some data analysts treat these data count as continuous measure and apply linear mixed effects model. But that practice ignores two facts: the data are really discrete, and the distributions of count variables are typically highly skewed. For these reasons, it may be inappropriate to use models that assume normally (multivariate) distributed errors. In this study, a Poisson regression model with normal random effects and a model that accounts for both correlations between repeated measures and over dispersion simultaneously, combined (Poisson-Gamma-Normal) model was considered in line with [8] and [9, 10].

\subsubsection{Data Exploration}

Before directly modeling a given data, it is important to make exploratory analysis to observe the structure and pattern of the data. Data exploration gives some direction to select the appropriate statistical model for a given data. In this study, individual profile plot, mean profile plot and variance structure were considered as parts of data exploration.

\subsubsection{Statistical Modeling}

The data of this study is longitudinal count data. As a result, subject specific (Poisson-Normal and Poisson-Gamma-Normal) models which include subject specific random effects were employed in the study by starting from standard count data models (Poisson and Poisson-Gamma models).

\section{Poisson-Normal-Model}

The generalized linear mixed model is the most frequently used random-effects model for non-Gaussian repeated measurements. It is straight-forward extension of the generalized linear model by addition of random effects in the model.

In generalized linear mixed models, conditionally on qdimensional random effects bi, the outcomes Yij are assumed to be independent with exponential-family densities of the form:

$$
\mathrm{f}_{\mathrm{ij}}\left(\mathrm{y}_{\mathrm{ij}} \mid \mathrm{b}_{\mathrm{i}}, \beta, \varphi\right)=\exp \left\{\varphi-1\left[\mathrm{y}_{\mathrm{ijj}} \theta_{\mathrm{ij}}-\Psi\left(\theta_{\mathrm{ij}}\right)\right]+\mathrm{C}\left(\mathrm{y}_{\mathrm{ij}}, \varphi\right)\right\}
$$

where, the random effects $b_{i}$, often assumed to be drawn independently from the $\mathrm{N}(0, \mathrm{D})$.

$\mathrm{D}$ is the variance-covariance matrix of the random effects.

$\theta_{\mathrm{ij}}=$ natural or canonical parameter.

$\Psi($.$) and C($.) are known functions.

$\mathrm{Y}_{\mathrm{ij}}=$ the value of the outcome variable for $\mathrm{i}^{\text {th }}$ individual at $\mathrm{j}^{\text {th }}$ time point.

$\beta=a$ p-dimensional vector of unknown fixed regression coefficients.

$b_{i}=a$ q-dimensional vector of unknown random regression coefficients for the $i^{\text {th }}$ individual.

$\varphi=$ a scale parameter

For $\mu_{\mathrm{ij}}=\mathrm{E}\left(\mathrm{Y}_{\mathrm{ij}} \mathrm{b}_{\mathrm{i}}\right)$ and known link function $\eta($.$) , the$ generalized linear mixed model can be expressed as:

$$
\eta\left(\mu_{\mathrm{ij}}\right)=\eta\left[E\left(Y_{\mathrm{ij}} \mid b_{\mathrm{i}}\right)\right]=X \mathrm{~T}_{\mathrm{ii}} \beta+\mathrm{ZT}_{\mathrm{ij}} \mathrm{b}_{\mathrm{i}}
$$

Where, $\mathrm{X}_{\mathrm{ij}}$ and $\mathrm{Z}_{\mathrm{ij}}$ are $\mathrm{p}$-dimensional and $\mathrm{q}$-dimensional vectors of known covariate values, respectively [9].

For the case of epilepsy which is Poisson data (i.e. $\mathrm{Y}_{\mathrm{ij}}$ $\left.\sim \operatorname{Poisson}\left(\lambda_{\mathrm{ij}}\right)\right)$, the generalized mixed Poisson model with normal random effects (Poisson-normal model) becomes

$$
\ln \left(\lambda_{\mathrm{ij}}\right)=\mathrm{XT}_{\mathrm{ii}} \beta+\mathrm{ZT}_{\mathrm{ij}} \mathrm{b}_{\mathrm{i}}
$$

This model is said to be Poisson-Normal model because it assumes Poisson distribution for the epilepsy data and normal distribution for the random effects $b_{i}$.

Overdispersion

Overdispersion in Poisson models occurs when the response variance is greater than the mean. Overdispersion is caused by correlation between individual responses. Overdispersion also arises when there are violations in the distributional assumptions of the data, such as when the data are clustered and thereby violate the likelihood independence of observations assumption. A model may be over dispersed if the value of the Pearson $\chi^{2}$ statistic divided by the degrees of freedom is greater than 1.0 [11]. It is clear that the Poisson distribution forces equality between mean and variance. However, comparing the sample average with the sample variance might reveal that this assumption is not true for a particular set of data. Likelihood Ratio Test can also be used to test overdispersion in count data.

One way to account for overdispersion in count data is through a two-stage approach. A commonly encountered instance is by assuming that $\mathrm{Y}_{\mathrm{i}} \mid \theta_{\mathrm{i}} \sim \operatorname{poi}\left(\theta_{\mathrm{i}} \lambda_{\mathrm{i}}\right)$.

Where $\theta_{\mathrm{i}}$ denote an independent and identically distributed (iid) sample of unit mean gamma random variables with shape parameter $\alpha(8)$. Conditional on $\theta \mathrm{i}$, the epileptic seizure of the $i^{i \text { th }}$ patient, $Y_{i}$ has a Poisson distribution with mean $\theta_{i} \lambda_{i}$.

The counts are then marginally independent negative binomial random variables $\left(\mathrm{Y}_{\mathrm{i}} \sim \mathrm{nb}\left(\alpha, \lambda_{\mathrm{i}}\right)\right)$ with mean $\lambda_{\mathrm{i}}$ and variance $\lambda_{\mathrm{i}}+$ $\lambda_{i}^{2} / \alpha$. Hence, the parameter $\alpha$ quantifies the amount of over dispersion with $\alpha=\infty$ corresponding to no overdispersion with respect to the Poisson distribution. The mass function of the negative binomial random variables is given by

$$
\operatorname{Pr}(Y i=y ; \alpha, \lambda \mathrm{i})=\frac{\Gamma(\mathrm{y}+\alpha)}{\Gamma(\alpha) y !}\left(\frac{\alpha}{\lambda+\alpha}\right) \alpha\left(\frac{\lambda i}{\lambda i+\alpha}\right) y
$$


The negative binomial model is given by $\log \left(\lambda_{\mathrm{i}}\right)=\mathrm{X}_{\mathrm{i}} \mathrm{T} \beta$.

Poisson-Gamma-Normal Model

A model combining the ideas from the Poisson Normal and overdispersion models for repeated Poisson data with overdispersion can be specified as follows $[9,10]$.

$$
\begin{gathered}
Y_{\mathrm{ij}} \sim \operatorname{poi}\left(\theta_{\mathrm{ij}} \lambda_{\mathrm{ij}}\right) \\
\lambda_{\mathrm{ij}}=\exp \left(\mathrm{X}_{\mathrm{ij}} \mathrm{T} \beta\right)+\mathrm{Z}_{\mathrm{ij}} \mathrm{Tb}_{\mathrm{i}}
\end{gathered}
$$

where $\theta_{\mathrm{ij}}$ capture overdispersion and denote an independent and identically distributed (iid) sample of unit mean gamma random variables with shape parameter $\alpha$ and scale parameter $\beta=1 / \alpha$.

$$
\mathrm{b}_{\mathrm{i}} \sim \mathrm{N}(0, \mathrm{D}) \text { and } \theta_{\mathrm{ij}} \sim \operatorname{Gamma}(\alpha, \beta)
$$

This model is called Poisson-Gamma-Normal (combined) model because it includes both normal $\left(b_{i}\right)$ and gamma $\left(\theta_{i j}\right)$ random effects to account for correlation and over dispersion respectively.

\section{Random Intercept only Model}

For the data with repeated follow up time, the effect of differences among the subjects (patients in this case) is expected to have significant influence on the event under study. The random intercept here is considered to see the between subject variability among patients not considering the effect of time. In fitting the random effect, estimating random intercept is used to test the intra class correlation among patients. This is because determining coefficients for all random intercept of patients are quit tedious.

\section{Random Intercept and Slope Model}

The introduction of random effects has important ramifications for the interpretation of the fixed-effects regression parameters. The random slope is used to test the between patients variability over time in that to study the difference among subject change in progressive of follow up time.

\subsubsection{Working Correlation Structures}

In modeling longitudinal data, it is important to specify the structure of correlation between the repeated measures of a subject. The most commonly used correlation structures include independence, exchangeable (compound symmetry) and autoregressive [11].

Independence correlation structure (IND): In this correlation structure observations are considered to be independent of one another. The structure assumes a zero correlation between subsequent measures of a subject within time points. This structure is useful if the size of panels is small and if there is evidently no time effect in the data. The scheme of this correlation structure can be shown as follows:

$$
\left(\begin{array}{lllllll}
1 & 0 & 0 & 0 & 0 & 0 & 0 \\
0 & 1 & 0 & 0 & 0 & 0 & 0 \\
0 & 0 & 1 & 0 & 0 & 0 & 0 \\
0 & 0 & 0 & 1 & 0 & 0 & 0 \\
0 & 0 & 0 & 0 & 1 & 0 & 0 \\
0 & 0 & 0 & 0 & 0 & 1 & 0 \\
0 & 0 & 0 & 0 & 0 & 0 & 1
\end{array}\right)
$$

Exchangeable correlation structure (Exch): The exchangeable correlation structure is the most commonly used structure. It is the default for several of the major commercial software implementations. The exchangeable correlation structure assumes that the correlations between measurements within time are the same, irrespective of any time interval. Any correlation value within the structure may be exchanged with any other hence the name exchangeable. The structure is as follows:

$$
\left(\begin{array}{lllllll}
1 & \rho & \rho & \rho & \rho & \rho & \rho \\
\rho & 1 & \rho & \rho & \rho & \rho & \rho \\
\rho & \rho & 1 & \rho & \rho & \rho & \rho \\
\rho & \rho & \rho & 1 & \rho & \rho & \rho \\
\rho & \rho & \rho & \rho & 1 & \rho & \rho \\
\rho & \rho & \rho & \rho & \rho & 1 & \rho \\
\rho & \rho & \rho & \rho & \rho & \rho & 1
\end{array}\right)
$$

Autoregressive order one correlation structure (AR(1)): The autoregressive $(\mathrm{AR}(1))$ correlation structure assumes that there is a marked decrease in correlation coefficient values with the corresponding increase in measurements within panel time intervals. Each off-diagonal from the main diagonal decreases by the square of the previous diagonal.

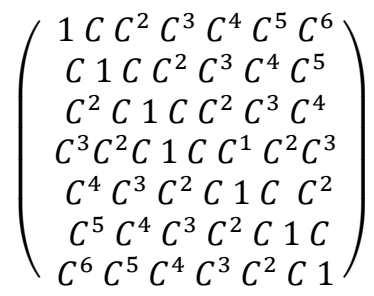

\subsubsection{Methods of Parameter Estimation}

Generalized linear mixed models (GLMMs) are a natural outgrowth of both linear mixed models and generalized linear models. GLMM's enable the accommodation of nonnormally distributed responses and specification of a possibly nonlinear link between the mean of the response and the predictors, and they can model overdispersion and correlation by incorporating random effects. Although there were computational problems to numerically evaluate the highdimensional integrals, maximum likelihood (ML) estimation will be used in this study.

Random-effects models can be fitted by maximization of the marginal likelihood, obtained by integrating out the random effects from conditional densities of the form:

$$
\mathrm{f}_{\mathrm{i}}\left(\mathrm{y}_{\mathrm{ij}} \mid \mathrm{b}_{\mathrm{i}}, \beta, \theta\right)=\exp \left\{\theta-1\left[\mathrm{y}_{\mathrm{ij}} \theta_{\mathrm{ij}}-\Psi\left(\theta_{\mathrm{ij}}\right)\right]+\mathrm{C}\left(\mathrm{y}_{\mathrm{ij}}, \theta\right)\right\}
$$

Therefore, as Poisson distribution is a member of exponential families, the likelihood contribution of patient $i$ for the Poisson-Normal model is:

$$
f_{i}\left(y_{i} \mid \beta, D, \theta\right)=\int \Pi f_{i j}\left(Y_{i j} \mid b_{i}, \beta, \theta\right) f\left(b_{i} \mid D\right) d b_{i}
$$

Where $\mathrm{D}$ is the variance-covariance matrix of the random effects. This implies that the likelihood function can be given as:

$$
\mathrm{L}(\beta, \mathrm{D}, \theta)=\Pi \mathrm{f}_{\mathrm{i}}\left(\mathrm{y}_{\mathrm{i}} \mid \beta, \mathrm{D}, \theta\right)=\Pi \int \Pi \mathrm{f}_{\mathrm{ij}}\left(\mathrm{Y}_{\mathrm{ij}} \mid \mathrm{b}_{\mathrm{i}}, \beta, \theta\right) \mathrm{f}\left(\mathrm{b}_{\mathrm{i}} \mid \mathrm{D}\right) \mathrm{db_{ \textrm {i } }}
$$


For the Poisson-Gamma-Normal or combined model the likelihood contribution of patient $\mathrm{i}$ and the likelihood

function can be given respectively as:

$$
\begin{gathered}
\mathrm{f}_{\mathrm{i}}\left(\mathrm{y}_{\mathrm{i}} \mid \beta, \mathrm{D}, \alpha, \beta^{*}\right)=\int \Pi \mathrm{f}_{\mathrm{ij}}\left(\mathrm{Y}_{\mathrm{ij}} \mid \mathrm{b}_{\mathrm{i}}, \beta, \theta_{\mathrm{i}}\right) \mathrm{f}\left(\mathrm{b}_{\mathrm{i}} \mid \mathrm{D}\right) \mathrm{f}\left(\theta_{\mathrm{i}} \mid \alpha, \beta\right) \mathrm{db} \mathrm{b}_{\mathrm{i}} \mathrm{d} \theta_{\mathrm{i}} \\
\mathrm{L}\left(\beta, \mathrm{D}, \alpha, \beta^{*}\right)=\Pi \mathrm{f}_{\mathrm{i}}\left(\mathrm{y}_{\mathrm{i}} \mid \beta, \mathrm{D}, \alpha, \beta^{*}\right)=\Pi \int \Pi \mathrm{f}_{\mathrm{ij}}\left(\mathrm{Y}_{\mathrm{ij}} \mid \mathrm{b}_{\mathrm{i}}, \beta, \theta_{\mathrm{i}}\right) \mathrm{f}\left(\mathrm{b}_{\mathrm{i}} \mid \mathrm{D}\right) \mathrm{f}\left(\theta_{\mathrm{i}} \mid \alpha, \beta^{*}\right) \mathrm{dbi} \theta_{\mathrm{i}}
\end{gathered}
$$

where $\beta$ is a p-dimensional vector of unknown fixed regression coefficients and $\beta *$ is the scale parameter of the gamma distribution. The key problem in maximizing both likelihood functions in equation (7) and in equation (9) is the presence of $n$ integrals over the random effects $b_{i}$ and $\theta_{i}$. To overcome this problem, different approximation methods to maximize the likelihood function have been proposed. These include numerical integration and series expansion methods, including penalized quasilikelihood and marginal quasilikelihood, Laplace approximation, adaptive Gaussian quadrature approximation, etc.

\subsubsection{Model and Variable Selection}

For all models, to select the important variables, first the main effect, main effect by time interaction and main effect by main effect interactions were incorporated to the initial candidate models and, then the highly non-significant interaction effects were removed and the models were refitted again and so on. i.e. Unautomated back ward selection technique was employed to select significant factors to be included in the final model. The best model that can fit the data was selected depending on different information criteria (AIC, BIC and -2loglikelihood). The model with smallest values of information criteria was selected as the best model to fit the data well.

\section{Results and Discussion}

\subsection{Descriptive Analysis}

A total of 337 epileptic seizure patients who were enrolled for treatment between January 1, 2016 to April 30, 2018 at Gondar Referral Hospital were included in this study. Among the 337 patients, $171(50.7 \%)$ were females and the remaining $166(49.3 \%)$ were males.

Table 2. Summary of epileptic seizure of patients at baseline and age.

\begin{tabular}{lllll}
\hline Variables & N & Mean & Std & Variance \\
\hline Baseline & 337 & 7.96 & 5.60 & 31.31 \\
Age & 337 & 29.10 & 9.40 & 88.36 \\
\hline
\end{tabular}

From Table 2 the average epileptic seizure of the patients at the start of the treatment was 7.96 with standard variation of 5.60. The data of the continuous covariate included in this study is summarized in Table 2 and showed that mean and standard deviation of ages of patients are 29.10 and 9.40 respectively.

Table 3. Summary of epileptic seizure for categorical Covariates.

\begin{tabular}{llll}
\hline Variables & Categories & Frequency & Percent \\
\hline \multirow{2}{*}{ Gender } & Female & 171 & 50.7 \\
& Male & 166 & 49.3 \\
\hline
\end{tabular}

\begin{tabular}{llll}
\hline Variables & Categories & Frequency & Percent \\
\hline \multirow{2}{*}{ Residence } & Rural & 164 & 48.6 \\
& Urban & 173 & 51.4 \\
Brain injury & No & 261 & 77.4 \\
Physical exercise & Yes & 76 & 22.6 \\
& Yes & 253 & 75.1 \\
Family history & No & 84 & 24.9 \\
& Yes & 248 & 73.6 \\
Treatment & Phenobarbital & 89 & 26.4 \\
& Phenytoin & 51 & 83 \\
& Others & 3 & 16 \\
& First visit & 337 & 1 \\
Time & Second visit & 337 & 21.8 \\
& Third visit & 337 & 21.8 \\
& Fourth visit & 303 & 21.8 \\
& Fifth visit & 156 & 19.6 \\
Educational & Sixth visit & 68 & 10.1 \\
& Seventh visit & 8 & 4.4 \\
& No formal education & 45 & 0.5 \\
& Secondary & 93 & 13.4 \\
& Above & 117 & 27.6 \\
& & 82 & 34.7 \\
& & & 24.3 \\
\hline
\end{tabular}

As presented in Table 3, among the 337 patients included in the study, 166 were males and 171 were females. Phenobarbital has high number of patients $(283(83 \%))$ as compared with the other treatments. Phenytoin takes the second place in number of patients $(57(16 \%))$ and Others has the smallest number of patients $(3(1 \%))$. This table also shows that the percentage of time visit of patients have been decreased over time which may reveals the curable of patients from their disease. The category of variables of epileptic seizure of patients like rural, patients with brain injury, who did physical exercise, with family history of epilepsy and patients with no formal education have lower count number as compared with their respective category.

\section{Exploratory Data Analysis \\ Exploring Individual Profiles}

Figure 1 depicts the individual profile plot of epileptic seizure of patients which sampled from the total population of this study. It is the plot of epileptic seizure of each patient over time. The plot provides some information on between patients epileptic seizure variability and illustrate that there is change in patients' epileptic seizure over time. Some individuals have high epileptic seizure and others have a slowly decreasing epileptic seizure over time. As one could easily see from the graph, there is considerably large difference in the intercepts of individual trajectories. Similarly, some trajectories were steeper while others were almost horizontal, indicating the possible variability in the slope of epileptic seizure. Therefore, because of the 
variability in the intercept and slope of trajectories, using a

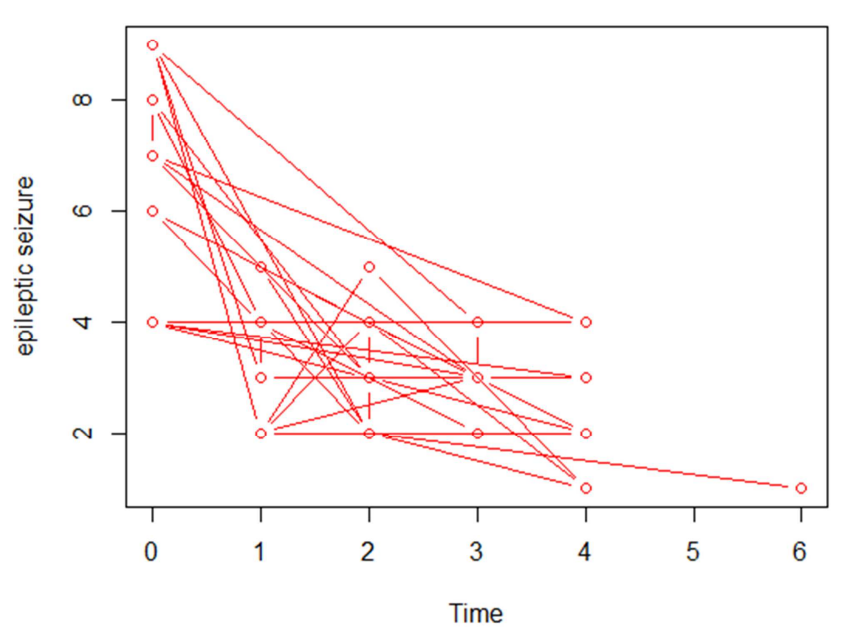

mixed model could fit the data very well.

Mean of epileptic seizure

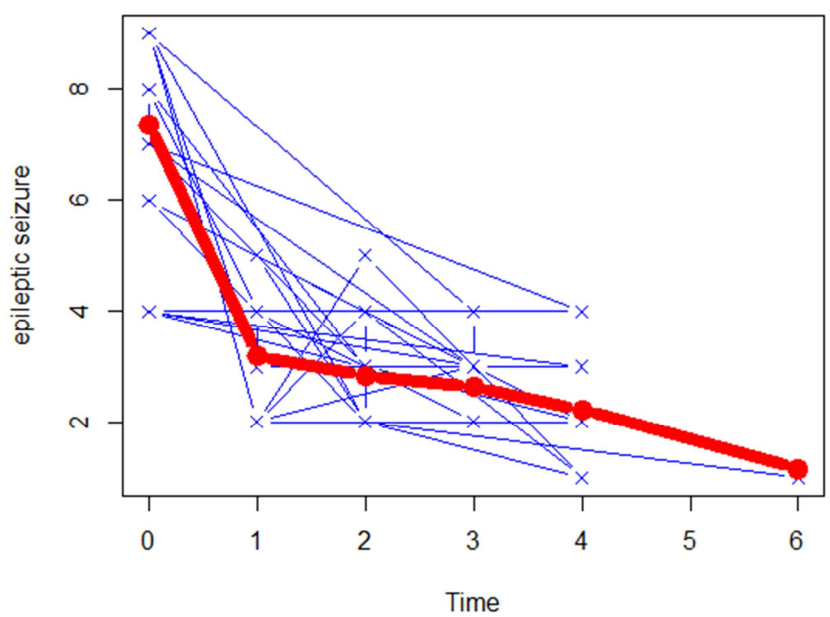

Figure 1. Individual profile plot for epileptic seizure of patients.

\section{Exploring Mean Profiles}

The overall mean profile plot of epileptic seizure shows a decreasing pattern of epileptic seizure over time (Figure 2). The mean epileptic seizure decreases in a high rate from baseline till the second visit and then it starts to decrease slowly from this point of time to the last.

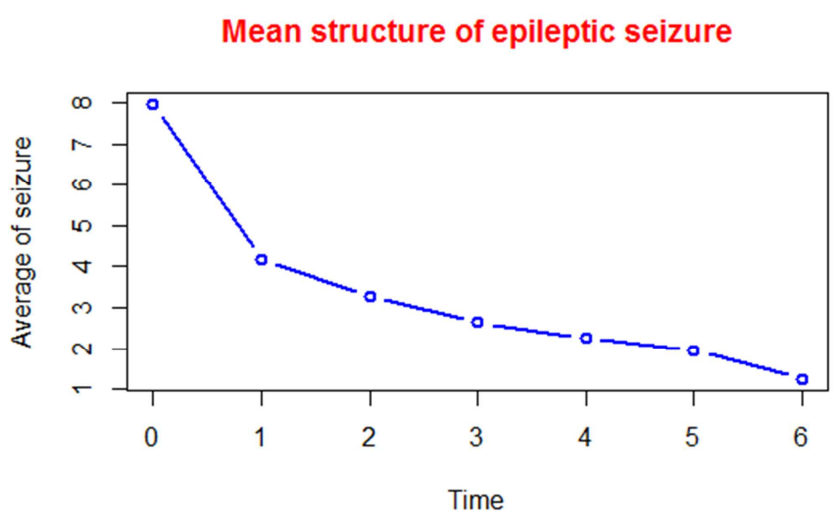

Figure 2. Mean profile plot of epileptic seizure of patients.

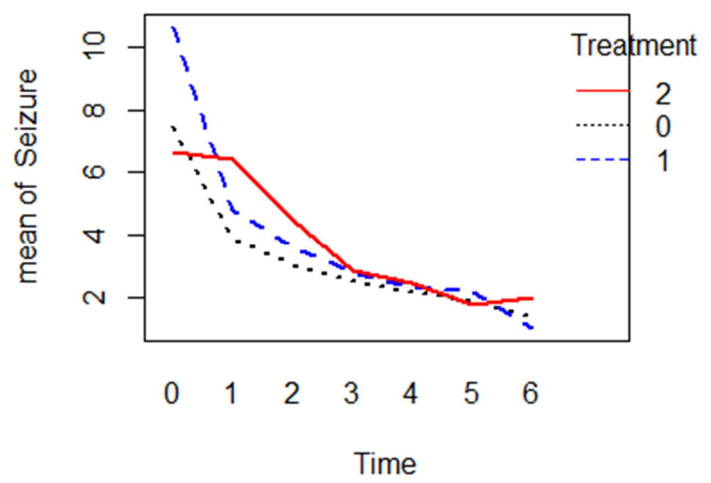

Figure 3. Mean profile plot of epileptic seizure by treatment.

As can be seen from Figure 3, the mean epileptic seizure profile of Phenobarbital is lower than the other treatments. Although the plot shows decreasing pattern on treatment group over time, it can be observed that the mean epileptic seizure of phenobarbital decreases at some time points and show a mild increases at another time points.

\section{Exploring variance structure}

The variance structure for epileptic seizure of patients shows an irregular pattern over time (Figure 4). It decreases at some point and increases at another point suggesting a non-constant variance.

\section{Variability in epileptic seizure over time}

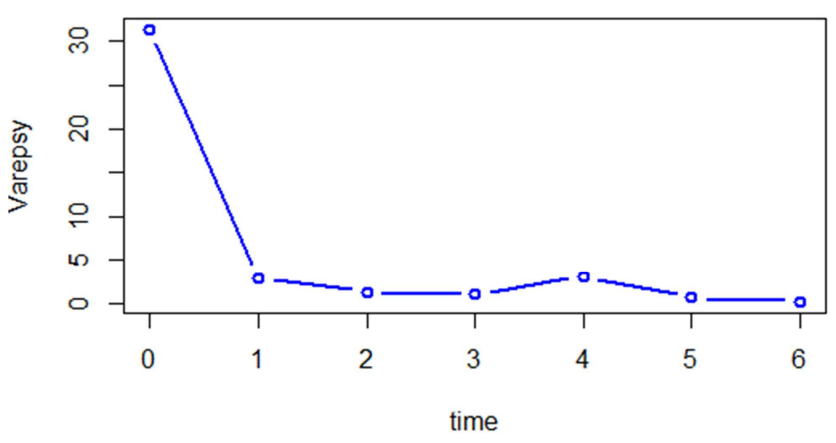

Figure 4. Variance structure of epileptic seizure of patients.

\subsection{Statistical Analysis Results}

This study included 337 epileptic seizures of patients who were under the treatment in Gondar Referral Hospital. The response variable of the study was epileptic seizure which is a count variable. Some of the candidate count data models were applied to fit epileptic seizure of patients and the final model was selected using different selection criteria. The candidate models employed to fit the epileptic seizure patients were Poisson, Negative Binomial, Poisson-Normal and Poisson-Gamma-Normal models.

Poisson and Poisson-Gamma Models

Generalized linear models are used for analyzing univariate non-Gaussian data. Poisson model is one of this 
models which is commonly used for the analysis of count data. Table 4 and Table 5 summarize the parameter estimates of fixed effects of Poisson and Poisson-gamma models employed on epileptic seizure.

It is clearly seen that there is an improvement in Poissongamma model with Akaike information criterion (AIC) value of 6251.61 as compared with the Poisson model of AIC
6369.89 displayed in Table 10.

A likelihood ratio (LR) test was used to test the null hypothesis that the restriction in the Poisson model is true. The test revealed that the null hypothesis is rejected with a chi-square test Statistic $=173.74(\mathrm{p}$-value $=<0.001)$. Table 5 displays the parameter estimates of the Poisson-gamma model along with standard errors.

Table 4. Parameter estimate of Poisson model.

\begin{tabular}{llllll}
\hline Parameter & Categories(if any) & Estimate & Standard Error & Wald Chi-Square & Pr $>$ ChiSq \\
\hline Intercept & & 2.239 & 0.087 & 658.07 & 0.000 \\
Gender & Female & -0.010 & 0.025 & 0.17 & 0.19 \\
Age & & 0.001 & 0.001 & 0.01 & 0.659 \\
Residence & Rural & -0.003 & 0.026 & 2.84 & 0.922 \\
Educational_level & No_formal_education & -0.074 & 0.044 & 0.092 \\
Educational_level & Primary & -0.070 & 0.036 & 3.83 & 0.050 \\
Educational_level & Secondary & -0.046 & 0.033 & 1.93 & 0.165 \\
Physical_exercise & No & 0.065 & 0.029 & 0.028 \\
Family_history & No & -0.017 & 0.028 & 0.38 \\
Brain_injury & No & -0.106 & 0.029 & 0.38 & 0.000 \\
Treatment & Phenobarbital & -0.241 & 0.061 & 12.98 & 0.000 \\
Treatment & Phenytoin & -0.031 & 0.063 & 0.24 & 0.621 \\
Time & & -1.355 & 0.010 & 17872.9 & 0.000 \\
\hline
\end{tabular}

Table 5. Parameter estimates of Poisson-gamma model.

\begin{tabular}{llllll}
\hline Parameter & Categories(if any) & Estimate & Standard Error & Wald Chi-Square & Pr $>$ ChiSq \\
\hline Intercept & & 2.227 & 0.098 & 513.05 & 0.000 \\
Gender & Female & -0.013 & 0.028 & 0.20 & 0.651 \\
Age & & 0.004 & 0.002 & 0.08 & 0.773 \\
Residence & Rural & -0.006 & 0.029 & 0.04 & 0.849 \\
Educational_level & No_formal_education & -0.069 & 0.049 & 2.87 & 0.161 \\
Educational_level & Primary & -0.068 & 0.041 & 0.093 \\
Educational_level & Secondary & -0.046 & 0.034 & 1.50 & 0.2212 \\
Physical_exercise & No & 0.057 & 0.033 & 3.00 & 0.083 \\
Family_history & No & -0.018 & 0.032 & 0.31 & 0.576 \\
Brain_injury & No & -0.098 & 0.034 & 8.54 & 0.004 \\
Treatment & Phenobarbital & -0.235 & 0.068 & 11.85 & 0.001 \\
Treatment & Phenytoin & -0.036 & 0.070 & 0.26 & 0.607 \\
Time & & -0.345 & 0.011 & 973.64 & 0.000 \\
\hline
\end{tabular}

Poisson-Normal and Poisson-Gamma-Normal Models

The Poisson-Normal model with only random intercept and both random intercept and slope were fitted using Laplace approximation method. Table 11 shows Poisson-Normal model with random intercept only was found to be the best fit as it has small information criteria values as compared with the one with both random intercept and slope. The parameter estimates of this model are displayed in Table 6 and Table 7. Depending on this model time, brain injury and treatment are found to be significant factors of epileptic seizure.

Table 6. Poisson-normal model with random intercept.

\begin{tabular}{lllllll}
\hline Effect & Categories(if any) & Estimate & Standard Error & DF & t Value & Pr $>|\mathbf{t}|$ \\
\hline Intercept & & 2.202 & 0.119 & 328 & 18.55 & 0.000 \\
Gender & Female & -0.009 & 0.037 & 1205 & -0.25 & 0.799 \\
Age & & 0.001 & 0.002 & 1205 & 0.55 & 0.580 \\
Residence & Rural & -0.007 & 0.038 & 1205 & -0.19 & 0.849 \\
Educational_level & No_formal_education & -0.060 & 0.064 & 1205 & -0.93 & 0.354 \\
Educational_level & Primary & -0.067 & 0.053 & 1205 & -1.26 & 0.207 \\
Educational_level & Secondary & -0.047 & 0.049 & 1205 & -0.96 & 0.339 \\
Physical_exercise & No & 0.054 & 0.043 & 1205 & 1.26 & 0.208 \\
Family_history & No & -0.015 & 0.042 & 1205 & -0.36 & 0.717 \\
Brain_injury & No & -0.099 & 0.044 & 1205 & -2.23 & 0.026 \\
\hline
\end{tabular}




\begin{tabular}{lllllll}
\hline Effect & Categories(if any) & Estimate & Standard Error & DF & t Value & Pr $>|\mathbf{t}|$ \\
\hline Treatment & Phenobarbital & -0.244 & 0.074 & 1205 & -3.29 & 0.001 \\
Treatment & Phenytoin & -0.048 & 0.074 & 1205 & -0.65 & 0.516 \\
Time & & -0.359 & 0.011 & 1205 & -34.36 & 0.000 \\
\hline
\end{tabular}

Table 7. Poisson-normal model with random intercept and slope.

\begin{tabular}{|c|c|c|c|c|c|c|}
\hline Effect & Categories(if any) & Estimate & Standard Error & DF & t Value & $\operatorname{Pr}>|\mathbf{t}|$ \\
\hline Intercept & & 2.239 & 0.091 & 1197 & 24.62 & 0.000 \\
\hline Gender & Female & -0.007 & 0.026 & 1197 & -0.25 & 0.802 \\
\hline Age & & 0.001 & 0.001 & 1197 & 0.56 & 0.576 \\
\hline Residence & Rural & 0.001 & 0.027 & 1197 & 0.00 & 0.996 \\
\hline Educational_level & No_formal_education & -0.074 & 0.045 & 1197 & -1.64 & 0.102 \\
\hline Educational_level & Primary & -0.070 & 0.037 & 1197 & -1.90 & 0.058 \\
\hline Educational_level & Secondary & -0.046 & 0.035 & 1197 & -1.33 & 0.184 \\
\hline Physical_exercise & No & 0.067 & 0.030 & 1197 & 2.20 & 0.028 \\
\hline Family_history & No & -0.018 & 0.029 & 1197 & -0.62 & 0.533 \\
\hline Brain_injury & No & -0.111 & 0.031 & 1197 & -3.61 & 0.000 \\
\hline Treatment & Phenobarbital & -0.246 & 0.065 & 1197 & -3.80 & 0.000 \\
\hline Treatment & Phenytoin & -0.028 & 0.067 & 1197 & -0.42 & 0.672 \\
\hline Time & & -0.363 & 0.011 & 336 & -32.29 & 0.000 \\
\hline
\end{tabular}

Table 8. Poisson-Gamma-Normal model with random intercept.

\begin{tabular}{|c|c|c|c|c|c|c|}
\hline Effect & Categories(if any) & Estimate & Standard Error & DF & t Value & $\operatorname{Pr}>|\mathbf{t}|$ \\
\hline Intercept & & 2.198 & 0.118 & 328 & 18.59 & 0.000 \\
\hline Gender & Female & -0.011 & 0.037 & 1205 & -0.30 & 0.761 \\
\hline Age & & 0.001 & 0.002 & 1205 & 0.49 & 0.626 \\
\hline Residence & Rural & -0.008 & 0.037 & 1205 & -0.22 & 0.825 \\
\hline Educational_level & No_formal_education & -0.059 & 0.063 & 1205 & -0.94 & 0.349 \\
\hline Educational_level & Primary & -0.067 & 0.052 & 1205 & -1.28 & 0.199 \\
\hline Educational_level & Secondary & -0.048 & 0.049 & 1205 & -0.98 & 0.326 \\
\hline Physical_exercise & No & 0.052 & 0.043 & 1205 & 1.22 & 0.221 \\
\hline Family_history & No & -0.014 & 0.041 & 1205 & -0.35 & 0.727 \\
\hline Brain_injury & No & -0.096 & 0.044 & 1205 & -2.20 & 0.028 \\
\hline Treatment & Phenobarbital & -0.241 & 0.076 & 1205 & -3.19 & 0.002 \\
\hline Treatment & Phenytoin & -0.051 & 0.075 & 1205 & -0.68 & 0.499 \\
\hline Time & & -0.351 & 0.011 & 1205 & -32.16 & 0.000 \\
\hline
\end{tabular}

Table 9. Poisson-Gamma-Normal Model with random intercept and slope.

\begin{tabular}{|c|c|c|c|c|c|c|}
\hline Effect & Categories(if any) & Estimate & Standard Error & DF & t Value & $\operatorname{Pr}>|\mathbf{t}|$ \\
\hline Intercept & & 2.227 & 0.099 & 1197 & 22.56 & 0.000 \\
\hline Gender & Female & -0.013 & 0.029 & 1197 & -0.44 & 0.662 \\
\hline Age & & 0.001 & 0.002 & 1197 & 0.30 & 0.765 \\
\hline Residence & Rural & -0.005 & 0.029 & 1197 & -0.18 & 0.858 \\
\hline Educational_level & No_formal_education & -0.070 & 0.049 & 1197 & -1.41 & 0.159 \\
\hline Educational_level & Primary & -0.069 & 0.041 & 1197 & -1.68 & 0.092 \\
\hline Educational_level & Secondary & -0.046 & 0.038 & 1197 & -1.22 & 0.221 \\
\hline Physical_exercise & No & 0.058 & 0.033 & 1197 & 1.74 & 0.082 \\
\hline Family_history & No & -0.018 & 0.032 & 1197 & -0.56 & 0.576 \\
\hline Brain_injury & No & -0.099 & 0.034 & 1197 & -2.92 & 0.004 \\
\hline Treatment & Phenobarbital & -0.236 & 0.069 & 1197 & -3.43 & 0.001 \\
\hline Treatment & Phenytoin & -0.036 & 0.071 & 1197 & -0.51 & 0.608 \\
\hline Time & & -0.346 & 0.012 & 336 & -29.15 & 0.000 \\
\hline
\end{tabular}

\subsection{Model Comparison}

Table 10. Summary of information criteria of Poisson and Poisson-gamma models.

\begin{tabular}{lll}
\hline \multirow{2}{*}{ Models } & Criteria & \\
\cline { 2 - 3 } & AIC & BIC \\
\hline Poisson & 6369.89 & 6439.35 \\
Poisson-gamma & 6251.61 & 6326.42 \\
\hline
\end{tabular}

Model selection was done based on AIC, BIC and -2loglikelihood. Poisson, Poisson-Gamma, Random intercept and slope Poisson-Normal, random intercept and slope PoissonGamma-Normal models were compared to select a best model that fits the data.

As it is seen from Table 10 Poisson-gamma model is the better model as compared with Poisson model.

As clearly shown in Table 11, the random intercept 
Poisson-Gamma-Normal Model is best fit because it has the lowest AIC (6176.04), BIC (6233.34) and -2loglikelihood (6146.04) as compared with the other models. Therefore, the final model to fit the data is random intercept PoissonGamma-Normal model.

Table 11. Summary of information criteria of different models.

\begin{tabular}{llll}
\hline Models & Criteria & \\
\hline & AIC & BIC & \\
\hline Poisson-normal with random intercept & 6198.25 & 6251.73 & -2Logliklehood \\
Poisson-normal with random intercept and slope & 6367.78 & 6421.26 & 6170.25 \\
Poisson-gamma-normal with random intercept & 6176.04 & 6233.34 & 6339.78 \\
Poisson-gamma-normal with random intercept and slope & 6253.58 & 6310.88 \\
\hline
\end{tabular}

Table 12 compares different correlation structures and independent correlation structures found to be best correlation structure for this study.

Table 12. Summary of information criteria of correlation structures.

\begin{tabular}{llll}
\hline Models & AIC & BIC & -2Loglikelihood \\
\hline CS & 6178.04 & 6239.16 & 6146.04 \\
IND & 6176.04 & 6233.34 & 6146.04 \\
AR(1) & 6178.04 & 6239.16 & 6146.04 \\
\hline
\end{tabular}

For the final model, to select the important variables, all possible interactions were incorporated to the initial candidate model and, then the non-significant interaction effects were removed and the model was refitted again.

Table 13. Parameter estimates of Poisson-gamma-normal model with interaction.

\begin{tabular}{|c|c|c|c|c|c|c|}
\hline Effect & Categories(if any) & Estimate & Standard Error & DF & t Value & $\operatorname{Pr}>|t|$ \\
\hline Intercept & & 2.251 & 0.085 & 335 & 26.35 & 0.000 \\
\hline Brain_injury & No & -0.152 & 0.053 & 1204 & -2.89 & 0.004 \\
\hline Treatment & Phenobarbital & -0.246 & 0.075 & 1204 & -3.27 & 0.001 \\
\hline Treatment & Phenytoin & -0.061 & 0.076 & 1204 & -0.81 & 0.419 \\
\hline Time & & -0.377 & 0.023 & 1204 & -16.56 & 0.000 \\
\hline Time*Residence & Time*Rural & -0.033 & 0.017 & 1204 & -2.00 & 0.045 \\
\hline
\end{tabular}

Based on the results obtained from the Poisson-GammaNormal model, brain injury, treatment, time, interaction of time with residence and interaction of time with brain injury were found to be significant factors of epileptic seizure of a patient.

For a given patient, keeping the random intercept and other covariates constant, a one unit increase in time decreases epileptic seizure by a multiplicative factor of $\mathrm{e}^{-}$ $0.377=0.686$. A patient without brain injured has epileptic seizure of $\mathrm{e}^{-0.152}=0.859$ times less of a patient with brain injured, holding other covariates and random intercept constant. Holding other covariates constant, a patient with Phenobarbital treatment has lower epileptic seizure with a multiplicative factor of $\mathrm{e}^{-0.246}=0.783$ as compared with a patient who took others treatment. Similarly, keeping other covariates constant, a patient with Phenytoin treatment has lower epileptic seizure with a multiplicative factor of $\mathrm{e}^{-}$ ${ }^{0.061}=0.941$ as compared with a patient who took others treatment.

Keeping other covariates constant, a patient who live in rural has epileptic seizure of $\mathrm{e}^{-0.033}=0.968$ times less of a patient who live in urban based on time effect. Keeping other covariates constant, a patient without brain injured has higher epileptic seizure of $\mathrm{e}^{0.054}=1.056$ times that of a patient with brain injured based on time effect.

\subsection{Discussion of Results}

The effects of factors on the epileptic seizure of patients who have taken treatment in Gondar Referral Hospital were assessed using longitudinal count models as the response variable of interest epileptic seizure is a count variable. The results of the summary statistics displayed in Table 2 revealed that the variance at baseline time point is high which indicates that there was high variation at the start of the treatment among the patients' of epileptic seizure. As summary statistics displayed in figure 3 the mean factors of epileptic seizure of patients seem to be minimized over time which indicates that the epileptic seizures of patients get decreases as time increase. As explained in the model selection part, different longitudinal count data models were applied to the data to determine the appropriate model to epileptic seizure and Poisson-Gamma-Normal model with a random intercept was found to be the best fit of the data. As it is displayed in Table 13 covariates time, brain injury, treatment, interaction of time with residence and interaction of time with brain injury were significant factors of epileptic seizure of patients.

This study revealed that a patient who has taken treatment phenobarbital has lower epileptic seizure as compared with a 
patient who has taken other treatments. This finding is in agreement with the studies done in United Sates of America [12] which revealed benefit of treatment on epileptic seizure of patients and starting treatment with phenobarbital is beneficial in recovering his or her epileptic seizure as compared with the one with other treatments.

This study revealed that patients with longer time on treatment have good recovery of epileptic seizure than that of patients with short duration on the treatment. The finding is in agreement with the finding of the study [13] which revealed that the decrease of epileptic seizure of the patients over time.

This study also revealed that patients with brain injury have high risk of epileptic seizure than those with no brain injury. This result is in agreement with the finding of the study [3] which revealed that brain injury increase epileptic seizure of patients.

The finding of this study is in agreement with the findings of the studies [14] and [15] which revealed that there were no significant associations of sex and residence with epileptic seizure of patients respectively. But, our finding is in disagreement with the finding of the study [16] which revealed association of age with epileptic seizure of patients.

\section{Conclusions and Recommendations}

\subsection{Conclusions}

This study used data from epileptic seizure patients' followed-up treatment in Gondar Referral Hospital from January 1, 2016 to April 30, 2018. The study was aimed to identify risk factors for the epileptic seizure of patients who have taken treatments and followed-up at Gondar Referral Hospital. Longitudinal count data models were used in the analysis and finally, Poisson gamma normal model was selected as a final model to fit the data based on different criteria.

The risk factors included in this study were gender, age, residence, educational level, physical exercise, family history, brain injury, treatment and time. Among these factors, duration on treatment (time), treatment, brain injury, interaction of time with brain injury and interaction of time with residence were found to be significant factors for epileptic seizure of the patients. The rest gender, age, residence, educational level, physical exercise, family history and all possible interactions were found to be insignificant on epileptic seizure of the patients.

Finally, minimization of epileptic seizure in response to treatment was observed, most of the patients were at decreased epileptic seizure when enrolled for treatment which might contribute to low epileptic seizure in patients.

\subsection{Recommendations}

Based on the study findings, the following recommendations are made for policy makers, community and researchers.

1) Patients are strongly recommended not to be discouraged and stay on the treatment which affects epileptic seizure negatively.

2) Being free from brain injury contributes a lot to epileptic seizure reduction so patients should understand this and refrain themselves from such victim area.

3) Further studies should be conducted on interaction of time with residence and brain injury of epileptic seizure patients and identify other factors that are not included in this study.

\section{Abbreviations}

$\begin{array}{ll}\text { AIC } & \text { Akaike information criteria } \\ \text { AED } & \text { Antiepileptic Drug } \\ \text { BIC } & \text { Bayesian information criteria } \\ \text { CBD } & \text { Cannabidiol } \\ \text { CI } & \text { Confidence interval } \\ \text { CSA } & \text { Central statistical agency } \\ \text { EEG } & \text { Electroencegarphic } \\ \text { FBH } & \text { Foundation for better health } \\ \text { GLMMs } & \text { Generalized linear mixed models } \\ \text { IBE } & \text { International bureau for epilepsy } \\ \text { ICES } & \text { International classification of epileptic seizure } \\ \text { ILAE } & \text { International league against epilepsy } \\ \text { ML } & \text { Maximum likelihood } \\ \text { NINDS } & \text { National institute of neurological disorder and } \\ \text { OR } & \text { stroke } \\ \text { TBI } & \text { Odds ratio } \\ \text { UN } & \text { United Nations } \\ \text { WHO } & \text { World health organization }\end{array}$

\section{References}

[1] Ngugi, A., Bottomley, C., Kleinschmidt, I., Sander, J., and Newton, C. (2010). Estimation of the burden of active and life - time epilepsy: A meta - analytic approach. Epilepsia, 51 (5), 883-890.

[2] Bazil C, Pedley T. (2005). Epilepsy. In: Rowland LP, editor. Merritt's Neurology. 11th ed. Philadelphia: Lippincott Williams \& Wilkins; pp. 990-1008.

[3] Lowenstein DH (2008). Seizures and epilepsy. In: Fauci A, Kasper DL, Longo DL, editors. Harrison's Principles of Internal Medicine. 17th ed. New York: McGraw-Hill; pp. 2498-2512. Section 2: Diseases of the Central Nervous System.

[4] Ayalew, M., and Muche, E. (2018). Patient reported adverse events among epileptic patients taking antiepileptic drugs. SAGE open medicine, 6, 2050312118772471.

[5] Berhanu, S., Alemu, S., Prevett, M., and Parry, E. (2009). Primary care treatment of epilepsy in rural Ethiopia: causes of default from follow-up. Seizure, 18 (2), 100-103.

[6] Megiddo, I., Colson, A., Chisholm, D., Dua, T., Nandi, A., and Laxminarayan, R. (2016). Health and economic benefits of public financing of epilepsy treatment in India: An agent based simulation model. Epilepsia, 57 (3), 464-474. 
[7] Verbeke, G. (1997). Linear mixed models for longitudinal data. In Linear mixed models in practice (pp. 63-153). Springer, New York, NY.

[8] Booth, J. G., Casella, G., Friedl, H., and Hobert, J. P. (2003). Negative binomial log linear mixed models. Statistical Modelling, 3 (3), 179-191.

[9] Molenberghs, G., Verbeke, G., and Demétrio, C. (2007). An extended random-effects approach to modeling repeated, overdispersed count data. Lifetime data analysis, 13 (4), 513-531.

[10] Molenberghs, G., Verbeke, G., Demétrio, C., and Vieira, A. M. (2010). A family of generalized linear models for repeated measures with normal and conjugate random effects. Statistical science, 25 (3), 325-347.

[11] Hilbe M. (2011). Negative Binomial Regression. Second edition. Cambridge University Press, New York.
[12] Devinsky, O., Marsh, E., Friedman, D., Thiele, E., Laux, L., Sullivan, J.,... and Wong, M. (2016). Cannabidiol in patients with treatment-resistant epilepsy: an open-label interventional trial. The Lancet Neurology, 15 (3), 270-278.

[13] Devinsky, O., Cross, J., Laux, L., Marsh, E., Miller, I., Nabbout, R.,... and Wright, S. (2017). Trial of cannabidiol for drug-resistant seizures in the Dravet syndrome. New England Journal of Medicine, 376 (21), 2011-2020.

[14] Jeffery (2008). Research paper on epilepsy." vol. 5, no. 9, article a022848.

[15] Gebre, A., and Haylay, A. (2018). Sociodemographic, Clinical Variables, and Quality of Life in Patients with Epilepsy in Mekelle City, Northern Ethiopia. Behavioural neurology, 2018.

[16] Shuo-BinJou, (2012). Epilepsy in the Elderly. International Journal of Gerontology Volume 6: Pages 63-67. 